\title{
High Resolution Imaging to Characterize the Structure and Biogeochemical Function of Microbial Biofilms
}

\author{
Matthew J. Marshall, Alice C. Dohnalkova, David W. Kennedy, Bruce W. Arey, and \\ James K. Frederickson
}

Pacific Northwest National Laboratory, Richland, WA 99354

Direct examination of natural and engineered environments has revealed that the majority of microorganisms in these systems live in structured communities termed biofilms. In addition to microbial cells, biofilms are comprised of a poorly characterized organic matrix commonly referred to as extracellular polymeric substance (EPS) that may play roles in facilitating microbial interactions and biogeochemical reactions including extracellular electron transfer. In our previous communications, we have examined the composition of EPS produced by Shewanella oneidensis MR-1 during uranium [U(VI)] biotransformation and the formation of reduced, extracellular uraninite [U(IV) $\mathrm{O}_{2}$ ] nanoparticles [1]. Using a combination of transmission electron microscopy (TEM), synchrotron-based, micro X-ray fluorescence ( $\mu$-XRF) imaging, and high-resolution immuno-TEM, a highly-hydrated $3 \mathrm{~d}$ bacterial EPS containing redox-active, extracellular electron transfer proteins was found to be produced during microbial metal reduction $[1,2]$. The juxtaposition of extracellular electron transfer proteins and nanoparticulate uraninite suggested that EPS played a key role in metal capture and precipitation and, possibly, extracellular electron transfer. Therefore, understanding how biofilm EPS functions and interacts with inorganic substrates such as metal ions and mineral surfaces connects the molecular-scale biogeochemical processes to those at the microorganism-level and provides insight to how microorganisms influence larger, pore-scale biogeochemical reactions.

Our current view of mineral-biofilm interactions has arisen from 2-dimensional images of 3-dimensional structures. Although a 3-dimensional structure of hydrated biofilm can readily be visualized using confocal laser scanning microscopy (CLSM), the relatively low resolution fails to resolve the fine scale interactions of EPS with minerals or newly formed nanoparticles (Fig.1). We have begun to employ cryogenic (cryo) sample processing to visualize specimens in the EM while fully preserved in their frozen-hydrated state. Preliminary experiments demonstrated that the cryoTEM produced dramatically improved cell architecture relative to the traditional TEM (Fig. 1). We have also begun to use cryoTEM to examine fully hydrated EPS. In these studies, we observed that EPS has an amorphous texture with low contrast due to sparse electron density. Notably, EPS visualization was enhanced in preliminary experiments where Shewanella cells were exposed to U(VI) (Fig. 1D). We speculate that addition of electron dense uranyl ions for bioreduction experiments provides a fortuitous contrasting agent for EPS; presumably due the binding of uranyl and/or U(IV) with EPS.

We have developed a protocol for cryo-preservation and cryoSEM visualization of biofilms [2]. Our preliminary experiments demonstrated that we can successfully cryopreserve relatively large pieces of biomass in a nearest-to-native, frozen-hydrated state. Our data suggested that the hydrated biofilm-associated EPS was significantly more extended than presumed (Fig. 2). We explored the underlying biofilm using the cryo- 
focused ion beam (cryoFIB) milling coupled with cryoSEM visualization. A cross-section taken perpendicular to the biofilm surface revealed bacteria with different degrees of mineralization and secondary mineral phases devoid of bacteria. Clearly, the use of cryoEM approaches to visualize hydrated structures such EPS will influence how we perceive microenvironments where the catalytic components within biofilm EPS interact with multivalent metal ions and influence subsurface biogeochemical processes.

This research was performed using the Environmental Molecular Sciences Laboratory (EMSL), a national scientific user facility sponsored by the DOE's Office of Biological and Environmental Research located at PNNL. Battelle Memorial Institute operates PNNL for the DOE under contract DE-AC05-76RL01830.

\section{References}

[1] M. J. Marshall et al. PLoS Biol 4 (2006) e268.

[2] A. C. Dohnalkova et al. Appl Environ Microbiol 77 (2011) 1262.
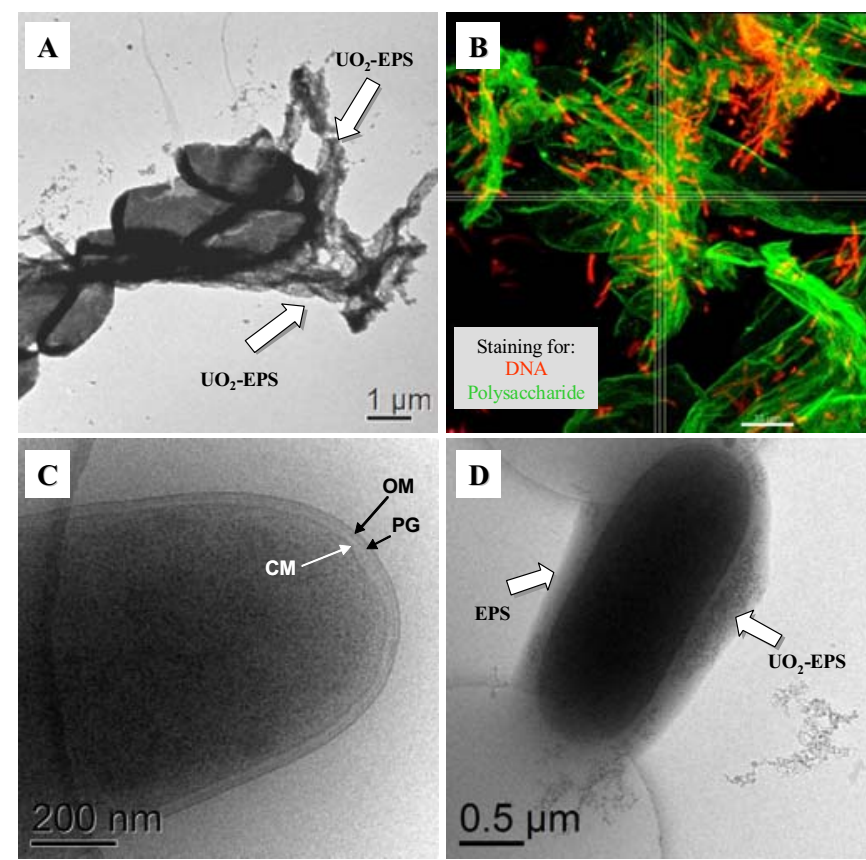

Fig. 1 Images of the 3-dimensional mineral-EPS interaction produced by $S$. oneidensis MR-1: (A) TEM of air dried whole mount showing EPS collapse; (B) hydrated EPS visualized using confocal laser scanning microscopy and EPSspecific stains; (C) cryoTEM of MR-1 cell illustrating enhanced visualization of outer and cell membranes (OM and $\mathrm{CM}$, respectively) and peptidoglycan layer (PG); (D) cryoTEM of MR-1 cell incubated with $\mathrm{U}(\mathrm{VI})$. The $\mathrm{U}$ enhanced visualization of EPS and mineral-EPS interaction are visible.

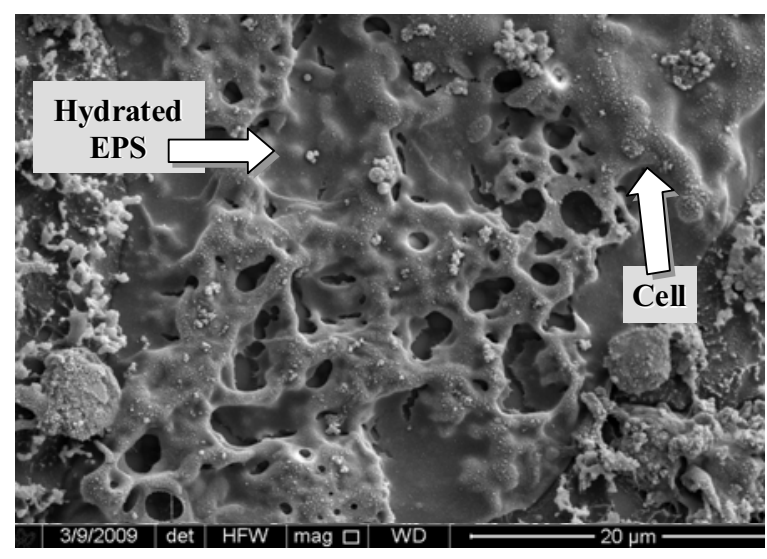

Fig. 2 CryoSEM micrograph at $-160^{\circ} \mathrm{C}$ showing a nearest-to-native state image of a mature biofilm produced during biostimulation activities at the Rifle IFRC. Cells and hydrated EPS are indicated with arrows. 